\title{
Interspecies Interactions Mediated by Technology: An Avian Case Study at the Zoo
}

\author{
Rébecca Kleinberger \\ MIT Media Lab \\ rebklein@media.mit.edu
}

Akito van Troyer

Berklee College of Music

avantroyer@berklee.edu

\author{
Anne H.K. Harrington \\ MIT \\ annekh@mit.edu
}

\author{
David Su \\ MIT Media Lab \\ david.d.su@gmail.com
}

\author{
Lydia Yu \\ MIT \\ lydiayu@mit.edu \\ Janet M. Baker
MIT Media Lab
janet_baker@email.com
}

Gabriel Miller
San Diego Zoo
gabemiller@gmail.com

\begin{abstract}
Enrichment is a methodology for caregivers to offer zoo animals improved psychological and physiological well-being. Although many species rely on auditory senses, sonic enrichment is rarely implemented. Zoo soundscapes are dominated by human-generated noises and do not respond meaningfully to animals' behavior. Designing interactive sonic enrichment systems for animals presents unique ergonomic, ethical, and agency-related challenges. We present a case study of such design. We deployed two novel interventions at the San Diego Zoo to allow Sampson, a music-savvy hyacinth macaw, to gain control over his sonic environment. Our results suggest that (1) the bird uses, understands, and benefits from the system, and (2) visitors play a major role in Sampson's engagement with this technology. With his new agency, the bird seemingly gains more control over his interactions with the public, creating an interspecies experience mediated by technology. The resulting animal-human-computer interaction may inform mediated interspecies experiences in the future.
\end{abstract}

\section{Author Keywords}

Enrichment; Sonic enrichment; Animal Music; Interspecies Interactions; Animal Computer Interaction; Animal Agency

\section{CCS Concepts}

-Human-centered computing $\rightarrow$ Human computer interaction (HCI); Haptic devices; User studies;

\section{INTRODUCTION}

Sampson is a solitary, 18-year-old hyacinth macaw (Anodorhynchus hyacinthinus) living at the San Diego Safari Park. According to his expert caregivers, the bird likes music and has

Permission to make digital or hard copies of all or part of this work for personal or classroom use is granted without fee provided that copies are not made or distributed for profit or commercial advantage and that copies bear this notice and the full citation on the first page. Copyrights for components of this work owned by others than the author(s) must be honored. Abstracting with credit is permitted. To copy otherwise, or republish, to post on servers or to redistribute to lists, requires prior specific permission and/or a fee. Request permissions from permissions@ acm.org.

CHI '20, April 25-30, 2020, Honolulu, HI, USA.

(C) 2020 Association for Computing Machinery.

ACM ISBN 978-1-4503-6708-0/20/04 _..\$15.00.

http://dx.doi.org/10.1145/3313831.3376858

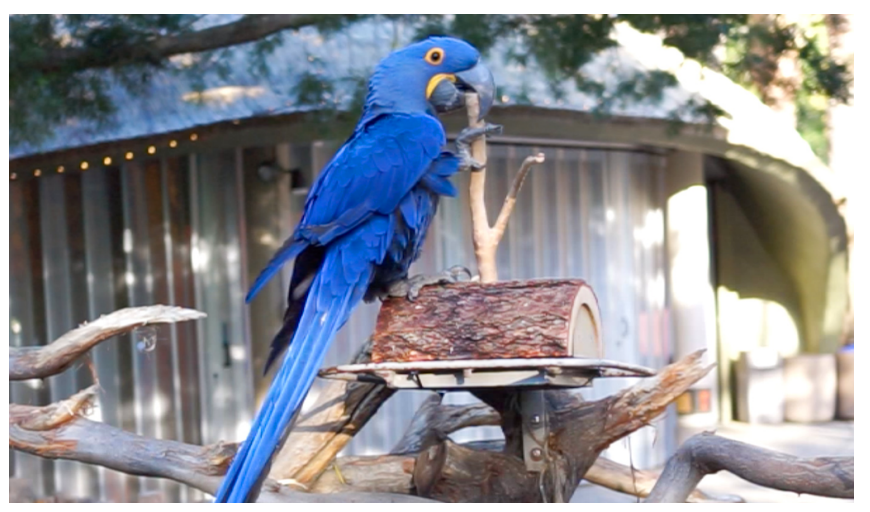

Figure 1. the Hyacinth Macaw Sampson interacting with the JoyBranch. The bird controls music by holding the stick with his foot and beak.

some favorite songs and specific musical tastes depending on his mood. The caregivers provide music for him occasionally during care sessions but would like to do so more frequently, and in ways that the bird can control himself. With the help of animal experts and zoo professionals, we designed and deployed two systems to allow Sampson to control and play music for and by himself from a series of five curated songs. Our design objectives were for our system to be ergonomic, ethical, engaging, understandable and agency-enhancing. The first intervention, JoyBranch, is a physical joystick embedded within a tree branch that the bird manipulates with his beak or feet to play a song. The second intervention, BobTrigger, relies on the bird's head bobbing - a natural behavior associated with positive engagement - as a visual cue to trigger music. We deployed and tested the two systems over five days. The presence of the experimenter and zoo visitors during the sessions revealed important factors regarding the bird's engagement with the system. We also collected comments and structured interviews from Samson's expert caregivers, Jenna Duarte and Michelle Handrus. This project was approved by the Zoo IACUC committee, which oversees the ethics and animal wellbeing during research.

Sampson's specific situation, in terms of location and personality, was central to the implementations and interpretation 


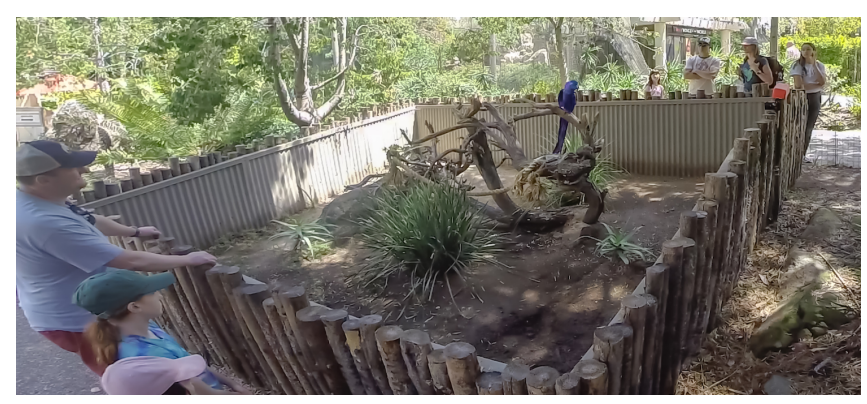

Figure 2. Sampson's enclosure is at the entrance of the zoo and visitors often stop by and interact with him.

of the results. His enclosure is about $4 \times 8 \mathrm{~m}$ surrounded by a waist-high fence (fig 2) preventing guests from approaching too closely. A large perch allows him to move freely within his enclosure. Every day, Sampson is brought in his enclosure at 7 am and back to his night chambers at $4 \mathrm{pm}$. As most parrots do [32], Sampson likes to "show off" by displaying his feathers, vocalizing, and parading in front of his keepers and visitors to attract their attention. He also expresses signs of excitement (head bobbing, head nodding, positive vocalizations) when seeing volunteers or patrons he knows well.

\section{BACKGROUND}

\section{Sonic Enrichment in Zoos}

From the 18th century human-focused menageries to the more animal-focused sanctuaries of today, zoos have evolved to respect and protect species [28]. Today, caregivers are trained and highly sensitive to the needs of the animals. Introduced in zoo husbandry in the 1940s, behavioral enrichment offers a framework for zoo professionals to enhance the quality of life of animals, enable them to express natural behaviors, and reduce boredom and stereotypic behaviors (abnormal behaviors frequently seen in captive animals) [63]. Enrichment techniques used today include enclosure design, toys, food delivery, adapted puzzles, co-housing different species, and sensory stimuli. Concerning sensory-based enrichment, Wells [60] considers that the greatest benefits for animal welfare are obtained when targeting the dominant sense of animals. Hearing is a dominant sense for many species, and many studies have evaluated the benefits of music_-including natural sounds [7], classical music [55, 19, 61], country music [55, 45, 54], and radio broadcasts $[3,25]$ —in enhancing the welfare of captive animals. However, most existing studies mainly focus on the effects of static recordings played and controlled by experimenters and thus do not provide insights into how sonic interactivity (i.e. customizing sounds based on responses from animals) might improve the efficacy of such sonic stimuli. In this project, we propose ways to tackle this gap in research by demonstrating instances of interactive sonic stimuli for a captive animal at the San Diego Safari Park. Kim-McCormack [27] shows the growing relevance of interactive digital applications for captive primates and insists on the importance of giving control to the animal. We believe that offering animals a similar kind of agency in shaping their sonic surroundings, especially in turning systems on or off, may yield substantial benefits. $74 \%$ of zoos surveyed by Hoy and colleagues never provide auditory enrichment, even though more than half of caregivers report that it is needed [24]. The few existing programs in sonic enrichment lack interactivity; for example, looped recordings of natural environments played through loudspeakers do not meaningfully respond to animals' attempts to interact. Agency can be defined as the propensity to engage actively with the environment with the main purpose of gathering knowledge and enhancing its skills [49]. The ability to interact seems indeed paramount in assuring realistic interaction and maximum benefit for the animal.

\section{Animal Music}

Previous work has also targeted animals' ability to produce music. Gupfinger presents a review of non-human musical expression [18] and proposes a phenomenology of animal music classifying instances into: Animal Movement as Control Source; Unconscious Performers; Trained Musicians; and Voluntary Musicians. We were inspired by their methodology for creating animal-centered musical interaction design, taking into account musical capacities as well as physical and cognitive abilities. Our objective differs as we are not trying to make the bird more musical, but to provide an enrichment system based on music and agency. Pons explores choice in sonic enrichment for orangutans in captivity by manipulating objects [43]. They use sounds (instead of human-made music) to allow them to create their own "music." This is an important step in the design of interactive enrichment, as it tackles the question of sonic agency for animals. Our designs and interventions further the work by testing and analyzing how an interactive auditory enrichment system performs in practice. French's work [14] on interactive auditory enrichment for elephants gives precedent for interventions with a physical trigger in non-primate captive animals. The "Sound Jam" workshop [15] demonstrates momentum within the ACI field to creating interactive auditory enrichment systems. Our work greatly benefits from the current climate of innovation in the field - a climate that is sensitive to issues of agency and ethics in animal technologies. Further motivating the need for interactiveness, Rivto found that there may be differences in how orangutans and humans experience music [46]. When given the choice, orangutans often chose silence over listening to sound. The animals exercise their right to choose not to have a sound played. Thus, interactive systems that give animals a choice in what they hear, as our system aims to do, is essential to creating an intervention that is truly enriching.

\section{Human-Animal Relationship (HAR) as enrichment}

Hosey lays the groundwork for understanding human-animal relationships in zoos and presents a model for understanding how past experiences with humans inform relationships in the present [22, 23]. His work strengthens our argument that it is necessary to consider the role of humans in the life of zoo animals especially during interventions. Indeed, human presence can often be detrimental to the wellbeing of animals in managed care. For instance, the presence of visitors has been shown to increase distress levels of wolves [42], pandas [44], orangutans [2], and koalas [30]. Anthropogenic noise pollution, such as construction noise, has also been shown to increase stress in (and reduce the health of) big cats [8] as well as laboratory, domestic, and free-living animals [26]. 
However, certain human-animal interactions offer possible benefits, especially for zoo animals. The uniqueness of each human-animal dyad can help explain the complexity of the connections between animals and their caregivers [57]. In her work, Claxton explores the effects of daily contact with familiar caregivers and unfamiliar visitors and concludes that interactions can lead to positive outcomes for the animals if intentionally designed as environmental enrichment [9]. She also highlights the importance of tailoring human contact on a species-by-species basis. Understanding interactions between animals and visitors can also help determine which characteristics and behaviors of visitors are most appealing to animals and lead to higher levels of animal-human interaction [12], playfulness [36], and energy expenditure [34]. In [41], Ig Nobel anthropology prize winner Persson shows that zoo chimpanzees imitate human visitors as often and as well as visitors imitate the chimpanzees. In this work, joint attention is key to a rich, mutual, cognitively beneficial multispecies shared experience. Other work also highlights the acute awareness of animals for the experimenters. Primates and birds have been shown to be seemingly aware of the impact of their actions on others' behavior [4] and are able to grasp the experimenter's intention [52] and engage socially [33,5]. Far from only serving as entertainment for the animals, such humananimal relationship (HAR) interactions appear to be related to general social and communicative needs.

Parrots are especially known for their need for interaction and attention, often interpreted as "showing off" [50, 40], Duarte reported that "Sampson loves to show off." To attract visitors, he will look at them, call, nod, bob, move back and forth in his enclosure, and flap his wings. "He wants compliments, he knows when people are praising him." Such performative behaviors reflect the importance of attention and companionship.

\section{The rival/model procedure}

The rich literature on parrot learning and intelligence, specifically from the work of pioneer Irene Pepperberg, further highlights the incredible social intelligence of birds, their awareness of the interspecies interactions in their surroundings, and their acute need for attention. Before her work, behaviorists mainly used Skinner-operant conditioning and had deemed it impossible to teach parrots how to talk, but by using the model/rival method developed by Todt in 1975 [53], Pepperberg was able to teach grey parrot Alex to identify and name more than 50 different objects and understand quantities up to six, among other abstract concepts [39]. The model/rival procedure used for training involves two people; one is the trainer and gives instructions, and the other is the model who gives correct and incorrect responses and acts as the student's rival for the trainer's attention. In some cases, the role of the rival can be played by another previously trained bird. The parrot, in the role of student, tries to reproduce the correct behavior motivated by gaining the attention of the human trainer [38]. This situation creates a triangular interaction between the bird, the trainer and the rival. The success of the model/rival procedures demonstrates the importance of the social context for parrots. In our case, we are less interested in training than in genuinely sparking the bird's interest and providing him with a playful tool and an experience that is intrinsically rewarding.
It might be impossible to truly separate intrinsic (pleasure) and extrinsic (human attention) rewards in this context. This historical approach in the context of teaching helps us to not only understand the complex role of the human relationship for the parrot, but also points to the importance of the sociological and psychological aspects underlying the human experience in the animal-computer-human triad.

\section{$\mathrm{ACl} / \mathrm{HCl}$}

Advances in technology can play an important role in creating enrichment systems for zoo animals. This need arises from the limited time that caregivers can spend with each animal [24]. However, we also believe that it is less desirable to provide a digital system for the animal to use in an isolated way than to design experiences that function in a social context while giving more control to the animals.

In terms of technological innovation, the field of animalcomputer interactions has produced inspiring previous work in solo enrichment systems. Technology for animals is a growing industry. Pets and livestock have inspired designers, artists and entrepreneurs to create new technologies and concepts, from fictional VR headsets for chickens [51] to off-the-shelf automatic feeders [56]. In the context of zoo-housed animals, computers and tablets have been used to provide primates with a variety of enriching applications, from face-matching games to "Tinder for orangutans" in breeding research $[10,47]$. In those examples, the main role of the human volunteers was to hold the devices. Keepers have expressed the need for enrichment systems that are "hands-off" as tablets often have to be held by caregivers, which is time-consuming and can cause safety issues. With the orangutans, frustration was observed because they were not able to hold the devices themselves [6].

However, some of the most interesting works involving animals and digital technologies are the ones that acknowledge the key role played by humans in animal environments. This statement is not to argue that animals do not have rich inner and outer lives independent from the human race, but when we introduce human technologies into their Ümwelt - their unique perception of the world - the human context should not be overlooked. Accordingly, there is a richness in exploring the junction where HCI and ACI meet [20,58]. The HCI community has proposed interesting instances of digital technologies used in the context of interspecies interactions between humans and non-human animals. Previous inspiring work from the HCI community involving animals has acknowledged and curated the human's role and behavior to appeal to the specific animal or species, from a challenging play partner $[35,62,64]$, to an empathetic audience [59], a conversational partner [29], or a provider of remote petting [31]. Such an approach has the potential to lead to higher levels of animalhuman interaction, playfulness, and energy expenditure [9] especially when the animal is provided with increased agency through the digitally mediated interspecies interaction.

\section{METHODS}

In this section, we first present our design choices, ideation process, and the design of our two systems (JoyBranch and BobTrigger) before going over elements of mapping and musical choices. Finally, we present the deployment methodology. 


\section{Approach and Design Choices}

Our design objectives were for our system to be ergonomic, ethical, engaging, understandable and to increase the agency of the bird. This project is shaped by existing enrichment practices, interviews, constraints, related work, and feedback from zoo professionals. Before designing and deploying the systems, we had extensive discussions and interviews with over ten Zoo professionals during a preliminary 1-week preparation trip to the Zoo. During that trip, we gathered information about the bird's needs and specific character from two bird experts working in the avian reproduction center, as well as from his caregivers, Handrus and Duarte. Handrus has been caring for Sampson everyday for over a year. Duarte has extensive expertise on parrots and has worked with Sampson for 5 years. We also met and presented our design for review to other animal experts who were involved in ideation and helped decide between design alternatives.

We had the chance to discuss our research with internationally renowned experts in this field such as Irene Pepperberg who gave us advice on how to use the rival/model method as an early priming mechanism to grasp the animal interest toward a device rather than using it for training. Four professionals working at the New England Exotic Wildlife Sanctuary, a parrot sanctuary also gave us feedback on how to understand bird attention, stress and engagement. Moderate physical activity, locomotion toward objects and physical interaction with toys and other branches can be used as metrics for engagement. Stress can be observed through pacing, feather plucking, and excessive grooming. Boredom manifests through long periods of inactivity and immobility. Focused staring may suggest attention and assessment of possible threats, while repeated gazing towards people suggests interest and intrigue. Such metrics were used in our methodology to assess the ethical and engaging aspects of our systems. Wenfei Tong, a researcher on bird vocalizations, recommended us to pay close attention to the bird's relationship ith researchers and the audience, which prompted us to record bird-visitor interactions and use gaze, vocalization, locomotion and body orientation of the bird and of the visitors to assess their engagement and level of rapport. David Rothenberg, musician and expert in interaction and in playing music with animals inspired us to think about the extent to which the visitors may play the role of an audience in motivating the bird's behavior.

All those discussions highly informed our design (interaction, twig-aspect, trigger, easy "off button," bob triggers) and methods used (shared audio space, human-bird-experimenter dynamics) as well as deciding which data to gather (continual video, $\log$ s) and how to conduct our protocol and analysis. This helped us tailor our design objectives toward a naturallooking object on which the bird has immediate agency based on physical contact/trigger and gesture that presents an easy "off button" by not interacting with it for 10 seconds. Those discussions also drove us into using interactive musical elements that create a shared auditory space in which to observe human-bird-experimenter social dynamics.

Duarte recommended using the bird's head bobbing to trigger music. When she plays Sampson music, the bird often bobs, and when she stopped the music the bird stops, looks at her and bobs a couple more times. She interprets this as the bird wanting her to play more: "he wants the music back". This might create a positive bias that needs to be acknowledged and assessed in future studies.

It should be noted that changes in the bird's environment are routine. Indeed, in accordance with the zoo rules, Sampson's environment is modified every few weeks and enriched with new branches, ropes and passive enrichment objects such as bells and wooden objects. Those were a source of inspiration for our design as we used the same texture, material and external aspect as those regular enrichment elements for our JoyBranch design.

\section{System Design}

Guided by those interactions with experts, we developed two interactive systems to potentially give Sampson control over his sonic environment. The first system is a physical device called the JoyBranch, which consists of a joystick mechanism embedded within a wooden $\log$ (fig 3 ) that was placed inside his enclosure. Each time the bird manipulates the joystick, music begins to play. When the stick is released, music continues for 10 seconds and then fades out linearly over 2 s (fig 6). For the second intervention, called BobTrigger, music is activated manually by an experimenter each time the bird bobs his head. If the bobbing stops for more than 10s, the music fades out linearly over $2 \mathrm{~s}$; if the bobbing is interrupted for less than 10 s and then restarted, the music stays on (fig 6). This is accomplished using a custom app.

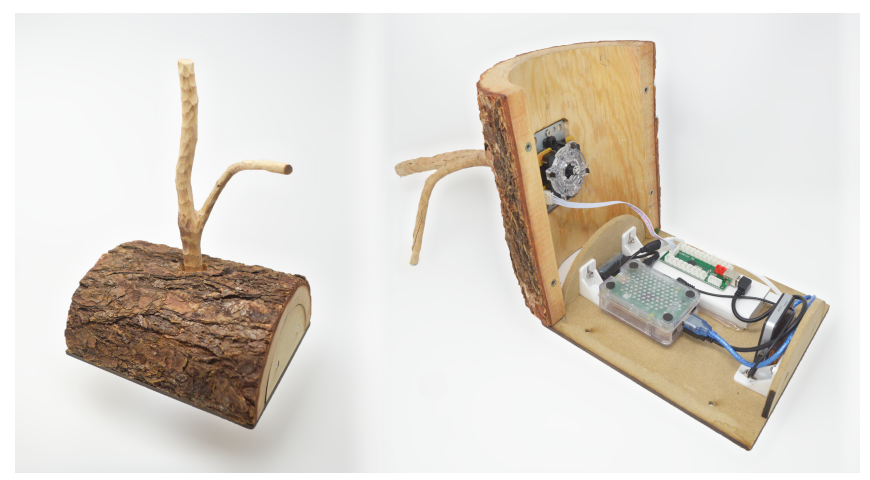

Figure 3. JoyBranch closed (left) and opened (right.)

The goal of the JoyBranch was to provide an interface to naturally entice the bird to interact and create a clear connection between his actions and the music activation. The interface design was inspired by existing low-tech enrichment techniques familiar to the bird (bells, ropes, wooden branches). The JoyBranch is designed to look as natural as possible: a section of a wooden $\log$ with a standard wooden perch attached. No electronics, sensors, cabling, or display screens are accessible or visible to the animal, and we used animal-grade wood designed for birds. Since parrots are very destructive, we designed our system to be breakable without endangering the bird. Inside the JoyBranch, the perch is attached to a joystick connected to an embedded Linux computer powered by a portable battery (fig 3 ). The bird only needs to push the stick five degrees to trigger the music, requiring only 1 newton of force. The audio output triggered by the movement of the 


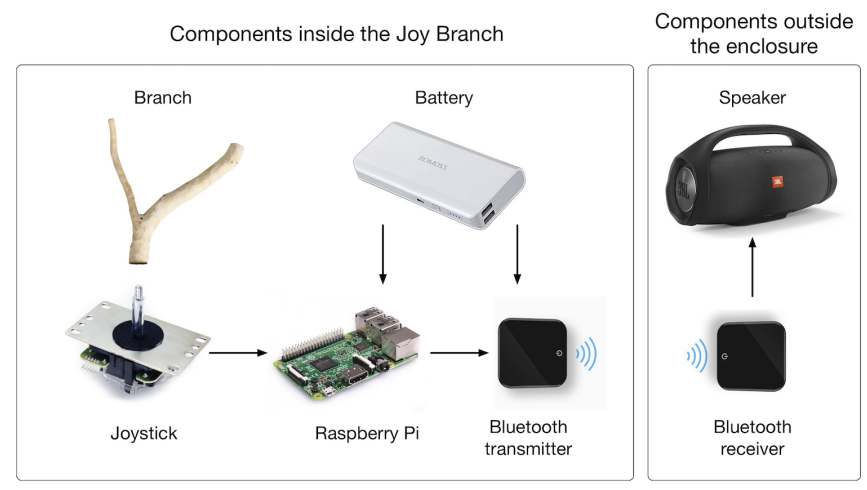

Figure 4. JoyBranch system diagram.

branch is sent by a Bluetooth transmitter to a receiver outside the enclosure. The sound is then played by a portable speaker. When the stick is released, it comes back to its neutral position. If the JoyBranch is not re-triggered within 10s, the music fades out. Within the enclosure, the JoyBranch is securely attached with zip ties to a permanent metal tray bolted to a perch in the enclosure. Sampson can approach the device from several angles while standing on nearby branches or the perch attached to the tray. The dimensions of the JoyBranch are designed to be ergonomic by matching Sampson's size and allowing him to hold the branch with his beak at different heights.

The BobTrigger intervention doesn't require the use of any additional elements in the enclosure and is engaging to the extent that it reinforces the bird bobbing behavior, commonly associated with positive engagement. Ethics are considered by only playing and maintaining the music when the bird actively bobs and expresses engagement. Although the bird has real agency over the music, understandability might be less obvious for the animal as the human in the loop is still technically in control and the relationship between Sampson's behavior and the music might be less clear for the bird.

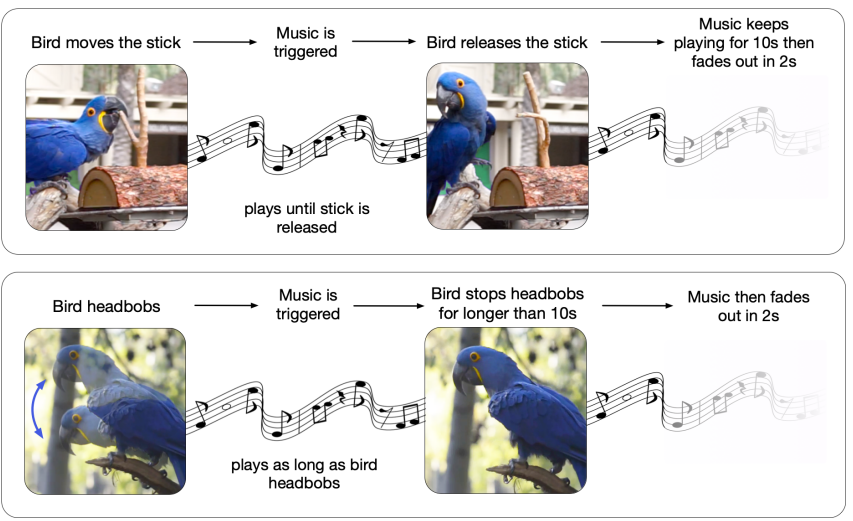

Figure 5. JoyBranch (top) \& BobTrigger (bottom) Interaction Design.

\section{Mapping}

For each session, we selected one of five songs. The choice of having the music fade out in the absence of interaction was to provide the bird with a metaphorical "off button," so the music would not play for long periods of time. This method was approved by the animal experts consulted. This choice has clear limitations, as the bird has to retrigger continuously if he wishes the music to continue, which might cause frustration if the music keeps fading out, but we believe that this trade-off is needed to assure an ethical experience.

The system was originally designed to allow the bird to choose between different musical tracks depending on the orientation of the joystick. However, for this first iteration, we started with the simplest mapping of only one track at the time regardless of the joystick orientation. Future work will explore more complex mappings.

The musical tracks used were five beat-heavy, up-tempo, popular dance songs: "Billie Jean" by Michael Jackson, "Karma Chameleon" by Culture Club, "I Like to Move It" by Erick Morillo, "Get Lucky" by Daft Punk, and "Jump In The Line" by Harry Belafonte. We rotated through the different tracks for every session, so the experience would not be too repetitive, but we used similar genres to limit the songs' influence on the bird. Sampson is already familiar with the genre and some of the songs and is known for engaging positively with such music when played by his keepers.

Parrots are often mentioned in research on animals and music, as they exhibit two key indicators for musicality in animal species: vocal learning and entrainment [21]. Entrainment involves the ability to synchronize movements to a beat [37]. It has been thought to be unique to species that can produce vocal mimicry (elephants, lovebirds, parrots, and in particular a famous sulfur-crested cockatoo named Snowball) but has also been observed in non-mimics such as sea lions [11]. When music is playing, Sampson sometimes exhibits entrainment through head-bobbing and head-whipping behaviors. Those behaviors are interpreted positively by his keepers. Previous work has explored the capability of parrots to generate musical content through interactive instruments [17] including a swing that creates a sound modulated by swinging and a joystick that produces single notes when triggered. Such work is important in understanding the complex musicality of the animals. In our case, we were more interested in reactions to complete upbeat songs.

The choice of using music instead of natural soundscapes containing bird sounds was motivated by ethical concerns. Indeed, there are ethical issues [48, 1] surrounding the use of so-called "audio playback" - the technique of playing back bird calls to engage birds - as it creates unfulfilled expectations of the presence of other birds, and may contain poorly understood bird calls that could have unexpected consequences.

\begin{tabular}{|l|l|l|}
\hline \multicolumn{1}{|c|}{ System } & \multicolumn{1}{c|}{ JoyBranch } & \multicolumn{1}{c|}{ BobTrigger } \\
\hline Ergonomy & $\begin{array}{l}\text { - Size \& Material } \\
\text { - Simple degrees of freedom } \\
\text { - Low force required }\end{array}$ & $\begin{array}{l}\text { - Natural bobbing behavior used } \\
\text { for trigger }\end{array}$ \\
\hline Ethics & $\begin{array}{l}\text { - Only plays if actively triggered } \\
\text { - Music stops if not re-triggered } \\
\text { - Short intervention time }\end{array}$ & $\begin{array}{l}\text { - Only plays if bobs } \\
\text { - Music stops if not re-triggered } \\
\text { - Short intervention time }\end{array}$ \\
\hline Engagement & $\begin{array}{l}\text { - Inspired by branch nibbling } \\
\text { - Need to be physically active } \\
\text { - No reward other than music }\end{array}$ & $\begin{array}{l}\text { - Based on a behavior of } \\
\text { positive engagement }\end{array}$ \\
\hline Understandability & $\begin{array}{l}\text { - Immediate relationship } \\
\text { between gesture and music }\end{array}$ & $\begin{array}{l}\text { - Emerges from previously } \\
\text { observed behavior of bobbing } \\
\text { when Samson wants music }\end{array}$ \\
\hline
\end{tabular}

Figure 6. Table of Design Objectives for JoyBranch and BobTrigger. 


\section{Deployment}

The evaluation aims to assess the enrichment potential for Sampson (frequency, understanding, ergonomy, agency) and the influence of visitors/experimenter on his use of the systems. Empirical methods used in the evaluation include interviews and observation. The testing lasted five consecutive days. On the morning of day 0 we ran three initial baseline sessions without any intervention. Then, from day 1 to day 4, we ran three sessions (one JoyBranch session and two BobTrigger sessions) each day between 6:30 am and $12 \mathrm{pm}$. We installed the JoyBranch on a tray with zip ties before 7 am, when Sampson is moved from his night enclosure to his exhibit. At 7 am, we ran a JoyBranch intervention session. At 9 am, we ran a first BobTrigger session (S2), and finally at 11 am we ran a second BobTrigger session (S3). The schedule was chosen to allow for at least an hour break between sessions. The sessions ran from 45 minutes to an hour each depending on the caregivers' schedule, who had to be present during the setup and breakdown. The resulting 16 sessions were observed by experimenters and facilitators and videotaped from two cameras at different angles for analysis and further inter-observer review.

On day 1, at the start of the initial JoyBranch intervention,we introduce the JoyBranch to Sampson through a 5-minute priming session. During this priming session, the experimenter showed Duarte how to interact with the system in front of Sampson. In doing so, we grasp the animal's interest toward the device and reassure the bird that the object is safe. For each BobTrigger intervention, the session was preceded by five seconds of music to announce the beginning of the session to the bird. In the analysis, we did not include times when Sampson's familiar caregivers were present because the bird is more naturally engaged and his attention is on the familiar faces in these situations. Moreover, we designed the two interventions specifically to enrich him during times when his keepers are not present.

\section{ANALYSIS}

\section{Tools and Labels}

To gather feedback on the animal's reaction to the interventions, we observed and labeled a series of different events, and behaviors during the baseline recordings, the JoyBranch interventions, and the BobTrigger interventions, using a semiassisted manual labeling interface [16]. From the videos and field notes, we labeled ten different observational behaviors from the bird: preening, feather puffing, stretching, standing on one leg, vocalizing, head bobbing, head nodding, head whips, eating, and moving within the enclosure. We also recorded instances of music being triggered and for how long. We labeled a total of 1,645 events and behaviors.

\section{Attention Labelling}

We collected information on the attention between visitors, Sampson and the experimenter/system through four metrics: bird attention toward the visitors (BAV), bird attention toward the experimenter (BAE), bird attention toward the JoyBranch (BAJB), and visitor attention toward the bird (VAB). To assess when the bird was focusing his attention toward the experimenter (BAE), we used field notes and video recordings and considered moments when the bird's head was oriented perpendicularly to the experimenter's location, his eye focused toward the experimenter with infrequent blinking, and he displayed little to no body motion or locomotion within the enclosure. We used the same metrics to assess when the bird was orienting his attention toward visitors (BAV). Contrary to the experimenter, who mainly remained still, visitors walk around the enclosure, and Sampson following them with his gaze is an additional indicator of his attention toward them. Because Sampson's enclosure is located at the entrance to the Safari Park, an average of 10 visitors per minute pass him, but not every visitor pays attention to him. To assess visitor attention toward the bird (VAB), we used field notes and video recordings to see when people approach the enclosure, interrupt their walk, look at the bird, and stay for at least a few seconds. We also used clues such as when the visitors take photos, talk with each other about the bird ("Look! A macaw!", "Look mama, the big bird!", etc.) or vocally address the bird directly ("Hi, macaw!", "Hello, bird," "You are a beautiful bird!", etc.) We collected a total of 851 moments of attention.

\section{Caregiver interviews}

The bird's caregivers Handrus and Duarte provided insight and help at all stages of the project. To not influence the bird during the interventions, they were not present during the sessions. We ran independent 1-hour long interview sessions with each of them. At the end of the last deployment day, 12 short 2-minute video clips of specific interesting behaviors from the sessions were shown to the caregivers. During the interviews, each clip was shown to the caregivers and they were asked 3 questions. For each clip, they were asked (1) to describe what they see, (2) whether they had seen this behavior before, and (3) how they would interpret this behavior. The recorded interviews were then transcribed into text and relevant information is presented in the Results section.

\section{RESULTS}

\section{Valence/Arousal map}

We analyzed and classified the diversity of Sampson's observable behaviors. Valence/arousal models for humans are used extensively in affective computing and HCI. Using previous research in the behavioral ecology of parrots, keeper feedback and interviews, as well as our own observations, we

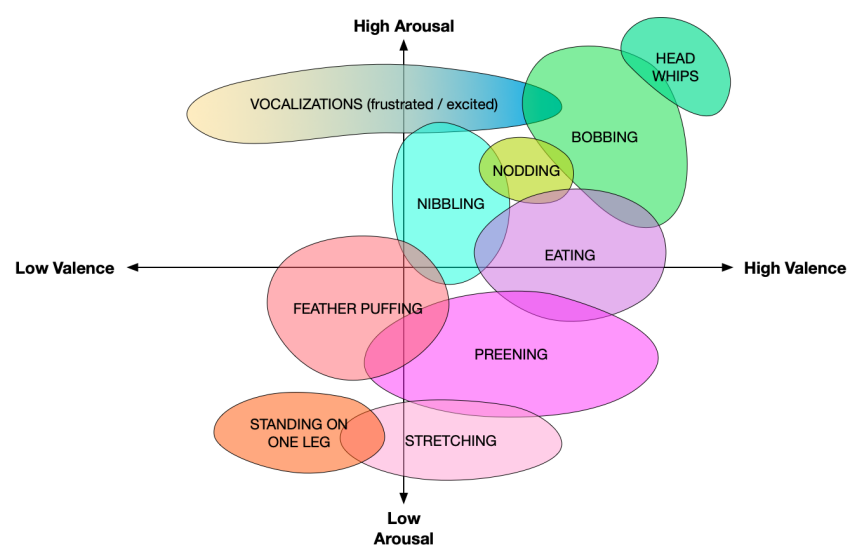

Figure 7. Arousal/valence map of Sampson's observational behaviors. 


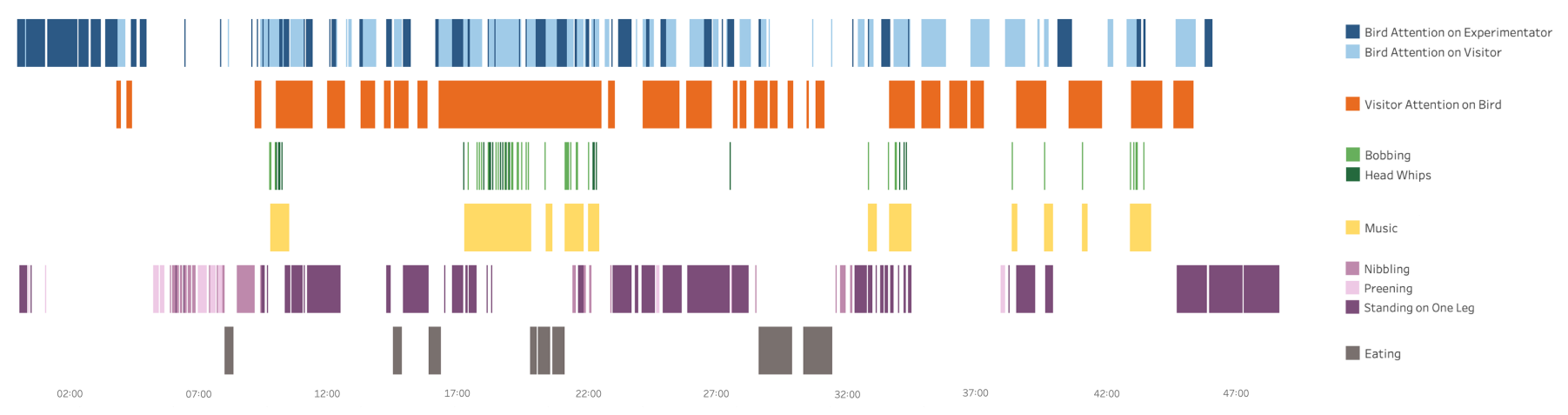

Figure 8. Representation of a typical example of behavioral correspondences during a session. (Day 2 Session 2 , duration approx 50m.)

established a behavioral valence/arousal map for Sampson. Preening and stretching are grooming behaviors that occur when the bird is calm and not deeply engaged with its environment (low arousal). A parrot would not self-groom if it were anxious about possible danger (medium/high valence). Feather puffing can have a range of meanings, from cold to frustration or anxiety. It is generally associated with slightly negative experiences (low/medium valence, variable arousal). Standing on one leg is a very low-energy activity - a restful pose that might indicate that the bird is tired (low arousal, medium/low valence). Eating sessions are positive and often medium-energetic. Parrots are very dexterous at peeling nuts with their beaks and tongues (medium arousal, high valence). Nibbling is a playful, vigorous activity consisting of chewing and rubbing the beak with a stick or branch. During the intervention, Sampson sometimes nibbled on regular branches and sometimes on the JoyBranch stick. Head nodding is a series of small, up-and-down head motions (high arousal) whereas bobbing is a more vigorous up-and-down motion that may include the whole body (very high arousal). Head whips are vigorous throws of the bird's head back and forth (highest arousal). All three head motions are seen as positive and suggest engagement with the music (high valence). Finally, vocalizing describes any sound the bird made. Both soft and strong vocalizations can express contentment and engagement, but in other cases, harsh vocalization can express frustration. Figure 7 represents Sampson's arousal/valence map of observational behaviors

\section{Session Overview}

In this section, we present a typical example of behaviors and suggest insights into some of the bird's behaviors (fig 8). The bird's attention is often brief and tends to alternate between the experimenter and the visitors. Visitor attention is more sustained. The correspondence between bobbing and music is causal; in this case, the music was triggered by the head bobs. We observe apparent non-co-occurrence between eating and resting/grooming behaviors (nibbling, preening, and standing on one leg). Bobbing behaviors tend to occur in groups and appear reinforced by music. Longer periods of visitor attention coincide with music-playing. In the following sections, we explore correlation and potential causality.

\section{Joy Branch Evaluation}

We were interested in assessing whether and how the bird used the systems. For the JoyBranch, we noted each time the bird triggered the music, for how long, and in which way. Figure 9 shows all the times the bird triggered the branch (either by poking at it, nibbling on it, holding it with his beak, or holding it with one foot) and the resulting intervals when the music plays. The third row shows every occurrence of head bobbing or head whips. The bird triggered the music a total of 33 times over $\mathrm{Z}$ days, for a total of $31 \mathrm{~m} 33 \mathrm{~s}$, representing $20 \%$ of the total session time. The bird used $1 / 5$ of his time interacting with the device, and the duration of each trigger got longer over time, from an average of $8 \mathrm{~s}$ on day 1 to an average of $2 \mathrm{~m} 26 \mathrm{~s}$ for the last day, which suggests increased interest (Fig 9).

Without instructions, the bird found four ways to interact with the branch. Initially, he only triggered the music by nibbling on the stick. Nibbling is a natural behavior, as attested by Handrus: "He turned it on, is also playing with it in his beak as he would normally with sticks." However, the time spent nibbling the JoyBranch (5\%) was higher than the time spent nibbling on passive sticks during baseline $(3.2 \%)$ or during BobTrigger sessions (1.15\%), suggesting a particular interest from the bird for the device. During the first day, Sampson nibbled on the branch, bobbed when the music plays, and once the music stopped, he would stop dancing and resume the nibbling and so on. It seemed that he hadn't yet made the

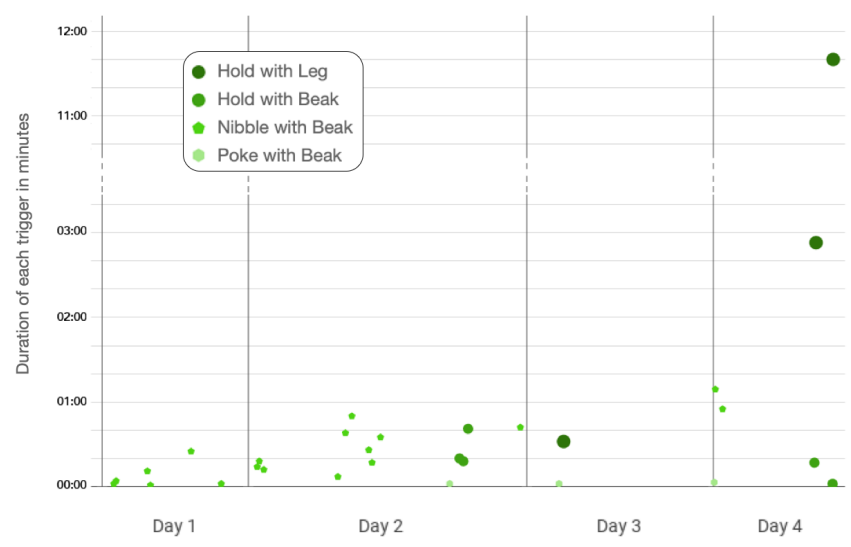

Figure 9. Day by day, the bird finds new ways to activate the JoyBranch, and triggers increase in duration, suggesting learning and exploration. 
connection between the music and his actions on the stick; according to Duarte: "I think he's just enjoying the music." However, during the second day, we observed instances when he simply poked the stick with his beak and then looked at the speaker and seemed to listen to the resulting music. Handrus interpreted this behavior as, "Wait!' Did I do that?" For Duarte, it can be seen as, " that speaker makes noise when I pull on the stick. Let's try it again and see if it does again." Then, he exhibited a novel behavior in holding the branch with his beak for extended amounts of time. Holding branches is uncommon for parrots, and neither Duarte nor Handrus had ever seen this behavior before: "He just went up and pulled it back. He didn't...go up to it,...trying to break it off. He didn't go up to it, and, rub his beak on it. He just...literally pulled it back. Like he knew that if he pulled it back, he just literally, pulled it back, which is just kind of weird." According to Duarte: "I think that he has figured it out. They don't generally do that." Indeed, macaws are very strong and enjoy breaking branches and nuts; however, both keepers noticed that the bird was being very gentle with the device: "He could just break it, normally if he wanted, he would break it very easily, but he doesn't want to. He is being very careful." On day 3, the bird continued holding the branch with his beak and then started holding it with his feet. Foot-holding behavior enabled him to also look around while the music was playing. The total time spent interacting with the device suggests a sustained interest in the JoyBranch, and newfound ways to interact with the branch day-by-day suggests learning, enjoyment, and interest. According to our observations and the caregiver's judgment, the bird understood the connection between the music and his actions on day 2 .

Bobbing always occurred during the music or shortly after, suggesting a strong connection between the two events as well as a positive experience for the bird. However, bobbing events became shorter and less frequent as the bird used more efficient ways to keep the music playing (longer nibbling, beak and foot holding). As parrots are thought to enjoy bobbing as a way to entrain to the music, we interpreted this trend as justifying the need for a no-contact, more ergonomic way to trigger the music. Additional considerations regarding novelty effect and possible adaptation of the bird to environment changes are explored in the Discussion.

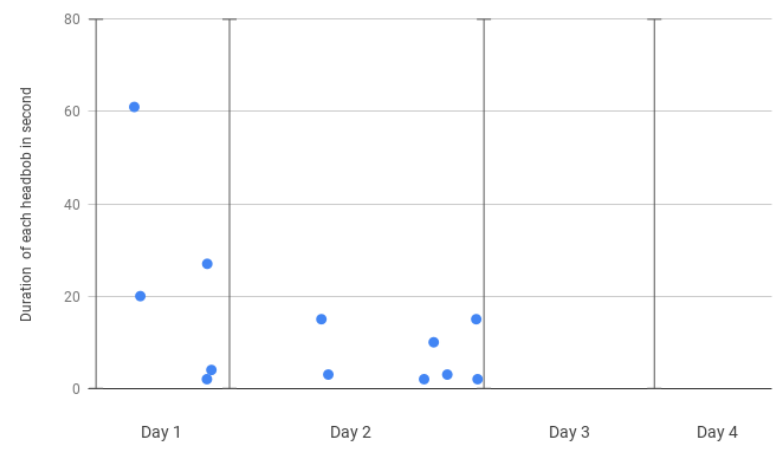

Figure 10. During the JoyBranch intervention, although occurrence and duration of triggers increase over time, bobbing become less frequent (0.18 occurrences of bobs per minute for day $1,0.13$ for day 2 , and 0 on day $3 \& 4$ ) and their duration becomes shorter with time. This might be due to the ergonomics of the branch as the bird finds more efficient ways to keep the music playing.

\section{Attention Analysis}

The data collected during the BobTrigger sessions give insight into (1) the use of the system, (2) the bird's perception of the intervention, and (3) the way he uses his new agency.

Contrary to the JoyBranch, the BobTrigger intervention doesn't involve a tangible object. Instead, each bob from the bird is observed, recorded by the experimenter and used to trigger music. Although the experimenter was not located directly next to the bird, Sampson noted their presence quickly and spent $19 \%$ of his time focused on the experimenter through short glimpses, at an average of 1.1 glimpses per minute.

It also appears that the bird quickly made the connection between the presence of the experimenter, bobbing, and the music. We distinguish headbob-triggers that occur while no music is playing, causing the music to start, versus entrained head bobs that occur while the music is already on. Within the eight intervention sessions, we recorded 94 instances of headbob-triggers, and in $73 \%$ of the cases (68 out of 94), the bird had his attention focused on the experimenter when triggering. We can also observe what can be interpreted as a learning curve for this phenomenon, as seen in Figure 11, which shows the evolution over the eight sessions.

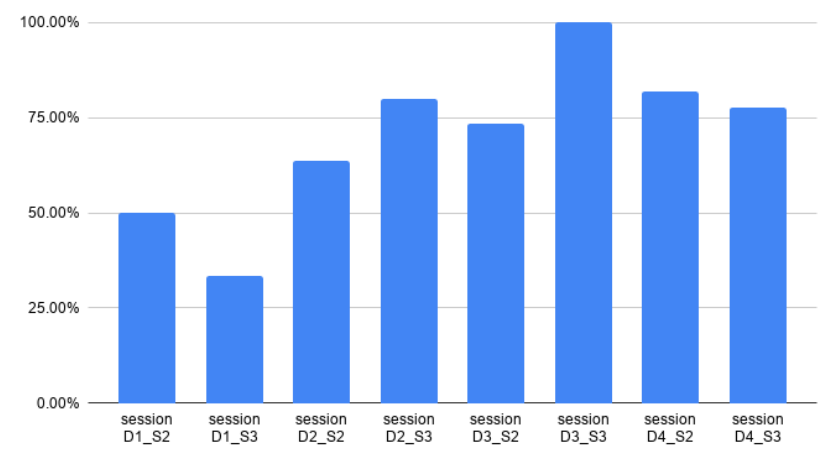

Figure 11. Over time, the bird more frequently looks at the experimenter when head-bobbing, suggesting a form of learning.

This trend suggests that the bird makes a connection between the human and the music. This might be interpreted as the bird assuming that the experimenter is in control and asking them for music. On the other hand, this could also indicate that, in order to gain the attention of the experimenter, the bird uses music to amplify his presence.

In addition, we observed 32 instances where the music was playing then stopped, then the bird looked at the experimenter and bobbed within 10 seconds of the music stopping, prompting the music to resume. This suggests that the bird understands the connection between bobs and music. In this context, instead of playing an invisible system, our results suggest the bird is actively "asking" the experimenter to play music. We could say that Sampson is "playing the experimenter" as an instrument to obtain the music. Indeed, once the music played, the bird generally stopped paying attention to the experimenter until the music stopped.

Zoo visitors represent an important component of Sampson's life. During baseline recordings, $47 \%$ of his time was spent 
observing and paying attention to the visitors. Although visitor attention is directed toward the bird about $51 \%$ of the time, only $22 \%$ of the total time is composed of mutual attention between visitors and the bird. This can be explained by several factors. The bird seems to have preferences for which visitors or groups of visitors he dedicates attention to (mainly children and frequent visitors). However, the bottleneck also comes from visitors, as they have the agency to walk away or direct attention elsewhere (e.g. by looking at signs or other exhibits) while the bird is focused on them. Indeed, during baseline, $80 \%$ of the instances of mutual attention (12 out of 15) ended with the visitors walking away while the bird was still looking at them.

To test whether the attention dynamic was modified by the technological intervention, we first compared the average duration of visitor attention toward the bird during baseline, then during the intervention while music was not playing, then when the music was playing. A Mann-Whitney U-test indicated that the duration of visitor attention was greater when the bird triggered music (mean=160s, std=107s) than when no music was playing (mean=32s, std=23s), $\mathrm{p}=3.3494 \mathrm{e}-17$.

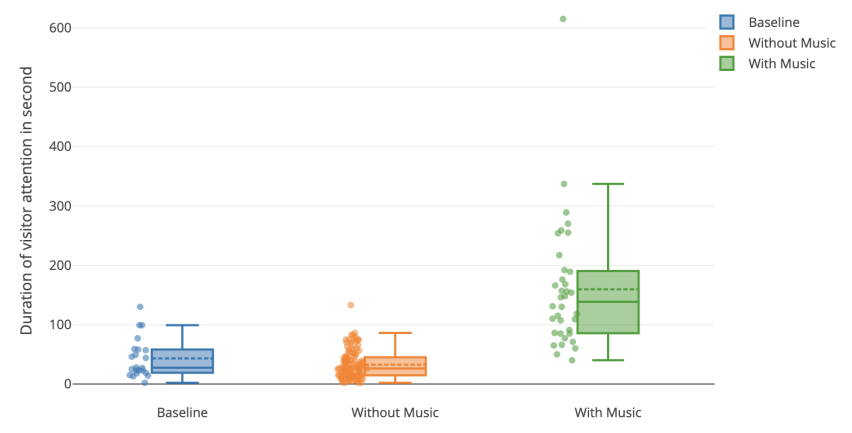

Figure 12. The average duration of visitor attention is comparable between baseline and when the bird doesn't trigger music during BobTrigger intervention. However, when the music is playing, the average duration of visitor attention is increased by a factor of 4 . This suggests that the bird might be using his new agency to control the visitor's attention.

In addition, the trigger of the music also influenced the distribution of mutual attention and timing dynamics. Indeed in the baseline, as stated above, in the baseline, $80 \%$ of mutual attention occurrences are ended by visitors. During the intervention, when no music is playing, the percentage of shared attention occurrences that ended by visitors while the bird is still paying attention at them is $63 \%$ (71 out of 112). This is comparable to the $80 \%$ during baseline. However, when the bird is playing music, this number drops to $25 \%$. This suggests that the bird has gained more control over the interaction and is now the one who decides when to end the interaction. Playing music is a successful way to keep the public interested.

\section{DISCUSSION}

\section{Future Work}

This preliminary analysis of the critical behaviors and correspondences observed through the intervention have brought insights into the perceived understanding and enjoyment of the animal and the influence of the experimenter and visitors.
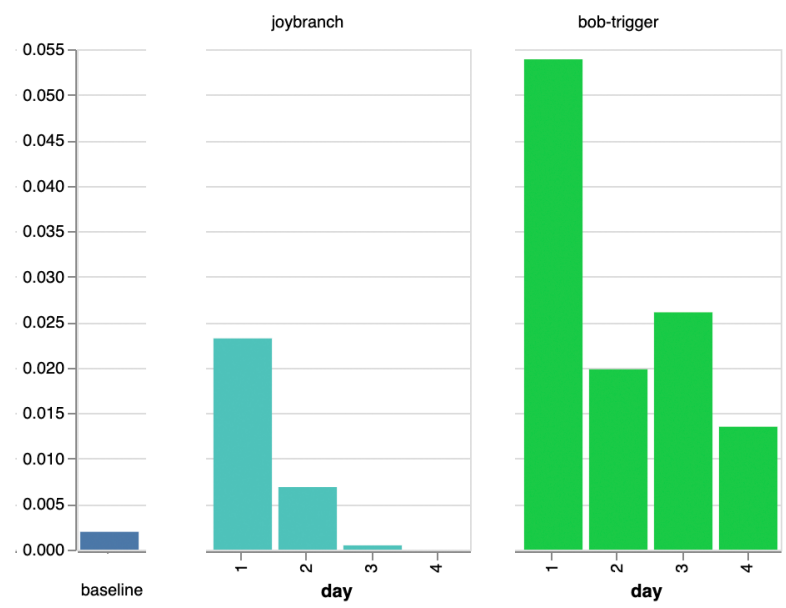

Figure 13 bobbing/nodding/whipping) for each intervention. Sampson triggered the music between 15 and $25 \%$ of the time by BobTrigger, and up to $45 \%$ of the time with the JoyBranch. During Day 3, JoyBranch usage was low - however, this was the session during which we remained out of sight for significant time.

In the future, ongoing work will provide a statistical understanding of the inter-dependencies of the various behaviors. Future iterations of the project would also need to take into account additional parameters that may influence the bird's and visitors' behavior (i.e. weather, temperature, precise time of day, what the bird ate, etc.) We know that Sampson tends to seek children's attention, but there may be other attributes of the visitors influencing his behaviors. In [12], experimenters noticed that primates were more likely to respond to men carrying objects. In other cases, visitor density and general noise were a key factor in the animal's responses [13].

This work also has clear limitations in the deployment to only one bird. Future work on a larger set of species and individuals is needed to generalize the results. However, the focused and personalized character of this work is what allowed us to take advantage of the individual experience of Sampson and take the time to engage in a dialogue with the animal. Opening up such a dialogue might be the most valuable outcome of the project for the researchers as well as, potentially, an inspiration for the bird to establish deeper contact.

The BobTrigger was based on the idea of using an automated system for bobbing recognition. Using the footage recorded during the deployment, we later implemented such an automated system that we will include in future deployments. However, this raises additional ethical questions as the experimenter was also monitoring the stress level of the bird. In addition, we believe, and in view of our results, that such a system should be deployed incorporating human collaboration. One possible future version involves visitors co-triggering music. To create an ultimate musical enrichment device for Sampson, we are working on a hybrid interface that incorporates the benefits of a physical system (tangibility, immediate connection action/sound) with the benefits of the deep-learning-based visually aware system to track the bird's behavior (no need to learn a new behavior, easy to deploy, fewer safety concerns). Such a system will also keep a log of the triggers and behaviors to provide more complete insights. 


\section{Novelty effect, attachment \& ethics}

We believe that the negative effects of introducing and removing interventions are limited. Indeed, from regular zoo policies, the bird is used to having novel objects regularly added and removed from the enclosure for enrichment and Sampson is also familiar to having music played for him by his caregivers. This hopefully limits the risks of Sampson to becoming overly attached to the device. In terms of ethics and companionship, Sampson is housed at night with other parrots with whom he routinely communicates from across the zoo. The zoo is very attentive to the question of animal companionship, which will be addressed in future deployments. During the intervention, we calibrated the music loudness such as not to add to current ambient $\mathrm{dB}$ (above existing human voices, ambient noise, announcements, etc). The possible implications, benefits, and risks of long-term deployment can be thoroughly explored through longitudinal testing.

\section{Implication for $\mathrm{HCl}$}

Our results suggest a relationship between attention dynamic and lead/follower influences in the triangle interactions between the bird, the experimenter, and the visitor. By gaining more control over his sonic environment, Sampson might have effectively gained more control over his interactions with humans. The interaction created became an interspecies experience mediated by technology.

This work offers insights for the field of HCI. By highlighting the experimenter's influence, this work supports the need for a human in the loop in ACI. This could also have potential implications if using technology to help visitors understand and read animal intent to lead to better interspecies understanding. Our design journey may also provide insights to future interactive technologies to engage humans and birds in zoos. The parallel use of physical vs gesture-driven systems for birds could expand the tangible vs virtual system in humans. In addition, our use of fully versus semi-automated systems broadens the discussion of such systems for humans. Our project also tackles the question of designing to understand and affect the attention ecology in zoos. Additionally, our approach also touches on the importance of animal involvement in interactive design. Finally, this work also may provide insight for more diverse HCI agents. Zoo visitors often wish to see interesting behaviors. Here, they can interact cooperatively with another species to play music. Our system enables novel interaction. It is an example of communication between different animals where the system itself becomes an agent to enrich the experience.

Anecdotal events during deployment also shed light on Sampson's personality. On day 3 , we wanted to see if the bird would use the JoyBranch if left alone. The experimenter went to hide and the zoo was not yet open, so there were no visitors. Not only did the bird show no interest in the device anymore, but he also started a long series of loud calls and repeated head rotations as if he were looking for the experimenter. This interpretation was confirmed by Duarte and Handrus: "I think he's surprised ... hey that girl's not here" and "Well, he's looking for you." This behavior lasted about five minutes until the bird walked to the JoyBranch and activated it while still calling.
After release, he resumed looking around. Once the music stopped, he then used his feet for the first time to hold the branch, allowing him to keep the music playing while looking around. The experimenter then came back from hiding, and when Sampson spotted her, he released the branch and stopped calling. More than a demonstration of a need to perform for an audience, this episode spoke to the bird's interests in companionship and establishment of rapport.

The bird's use of the device suggests that he may combine the simple enjoyment of listening to music with a more elaborate schema to attract and maintain public attention. The device seems to be used as a means to an end, and the bird's agency appears to be sometimes directed toward careful control of the visitors' and experimenter's attention.

In a more distant future, as more species facing extinction can only be conserved in managed care, we wish for zoos to integrate animal agency in all aspects of their design. We hope for a zoo of the future where a better balance might be established between animals and human-animals. Indeed, until animals can be the lead of co-creative projects with humans, we will remain egocentric in our view of ACI. If, however, we can reach the point where animals create their own understanding of all the parts of a human system, we can then truly start envisioning a meaningful interspecies internet.

\section{CONCLUSION}

We created naturalistic interactive systems for a solitary musicsavvy macaw to gain control over his sonic environment. Very few past instances of enrichment interventions have targeted the animal's sonic environment, even though for most species, auditory input is a major way in perceiving the world. The need for technological devices arises from the limited time caregivers can spend with each animal [24]. The deployment of the two interventions brought insight into the acute social awareness of the animal and the triangular interaction between the experimenter, the bird, and the visitors. Our results suggest not only that the bird understands, enjoys, and makes use of the systems, but also that the visitors play a major role in the animal's motivations and engagement with the technology. By gaining more control over his sonic environment, Sampson effectively gained more control over his interaction with the public. The interaction created became an interspecies experience mediated by technology. The resulting triangle interaction between the animal, the visitors, and the computer may bring insights into the potential of technology for future interspecies enrichment and communication.

\section{ACKNOWLEDGMENTS}

First and foremost, we thank Sampson for his participation. We also thank Michelle Handrus and Jenna Duarte for their extensive support. All procedures were approved by the San Diego Zoological Society IACUC (proposal 19-002)

\section{REFERENCES}

[1] American Birding Association. 2012. Code of ethics. http://www. aba.com

[2] L Birke. 2002. Effects of browse, human visitors and noise on the behaviour of captive orang utans. 
[3] L Brent and others. 1996. Physiological \& behavioral effects of radio music on singly housed baboons.

[4] Richard W Byrne and others. 2010. Primate social cognition: uniquely primate, uniquely social, or just unique?

[5] Malinda Carpenter and Josep Call. 2009. Comparing the imitative skills of children and nonhuman apes.

[6] Marcus Carter and others. 2015. Naturalism and ACI: augmenting zoo enclosures with digital technology. In $A C E$.

[7] AS Chamove. 1989. Cage design reduces emotionality in mice.

[8] Julia Chosy and others. 2014. Behavioral and physiological responses in felids to exhibit construction.

[9] Anna M Claxton. 2011. The potential of the human-animal relationship as an environmental enrichment for the welfare of zoo-housed animals.

[10] Andrea W Clay and others. 2011. The use of technology to enhance zoological parks.

[11] Peter Cook and others. 2013. A California sea lion can keep the beat: motor entrainment to rhythmic auditory stimuli in a non vocal mimic.

[12] Shelley Cook. 1995. Interaction sequences between chimpanzees and human visitors at the zoo.

[13] Alexandra Farrand. 2007. The effect of zoo visitors on the behaviour and welfare of zoo mammals.

[14] Fiona French and others. 2018a. High tech cognitive and acoustic enrichment for captive elephants.

[15] Fiona French and others. 2018b. SoundJam: acoustic design for auditory enrichment.

[16] Olivier Friard and others. 2016. BORIS: a free, versatile open-source event-logging software for video/audio coding and live observations.

[17] Reinhard Gupfinger and others. 2017. Sonic Experiments with Grey Parrots: A Report on Testing the Auditory Skills and Musical Preferences of Grey Parrots in Captivity.

[18] Reinhard Gupfinger and others. 2018. Animals make music: A look at non-human musical expression.

[19] G Gvaryahu and others. 1989. Filial imprinting, environmental enrichment, and music application effects on behavior and performance of meat strain chicks.

[20] Ilyena Hirskyj-Douglas and others. 2016. Where HCI meets ACI. In Proceedings of the 9th Nordic Conference on Human-Computer Interaction. ACM.

[21] Marisa Hoeschele and others. 2015. Searching for the origins of musicality across species.

[22] Geoff Hosey. 2008. A preliminary model of human-animal relationships in the zoo, 105-127.
[23] Geoff Hosey and others. 2015. Are we ignoring neutral and negative human-animal relationships in zoos?

[24] Julia M Hoy and others. 2010. Thirty years later: Enrichment practices for captive mammals.

[25] RB Jones. 2004. EnvironmentaI enrichment: the need for practical strategies to improve pouItry welfare, 215.

[26] Caitlin R Kight and John P Swaddle. 2011. How and why environmental noise impacts animals: an integrative, mechanistic review.

[27] Nicky NE Kim-McCormack and others. 2016. Is interactive technology a relevant and effective enrichment for captive great apes?

[28] Vernon N Kisling. 2000. Zoo and aquarium history: Ancient animal collections to zoological gardens. CRC.

[29] Donghyeon Ko and others. 2018. BubbleTalk: Enriching Experience with Fish by Supporting Human Behavior. In DIS'18. ACM.

[30] Megan J Larsen and others. 2014. Number of nearby visitors and noise level affect vigilance in captive koalas.

[31] Ping Lee and others. 2006. A mobile pet wearable computer and mixed reality system for human-poultry interaction through the internet.

[32] Andrew U Luescher. 2006. Manual of parrot behavior.

[33] Mark Nielsen. 2009. The imitative behaviour of children and chimpanzees: A window on the transmission of cultural traditions.

[34] AJ Nimon and others. 1992. Cross-species interaction and communication: a study method applied to captive siamang and long-billed corella contacts with humans.

[35] Frank Noz and Jinsoo An. 2011. Cat cat revolution: an interspecies gaming experience. In CHI' 11 . ACM.

[36] C Owen. 2004. Do visitors affect the Asian short-clawed otter in a captive environment. In Proceedings of the 6th Annual Symposium on Zoo Research-BIAZA. 202-211.

[37] Aniruddh D Patel and others. 2009. Experimental evidence for synchronization to a musical beat in a nonhuman animal.

[38] Irene M Pepperberg. 2002. Cognitive and communicative abilities of grey parrots.

[39] Irene M Pepperberg. 2009. The Alex studies: cognitive and communicative abilities of grey parrots.

[40] Irene M Pepperberg and Mary A McLaughlin. 1996. Effect of avian-human joint attention in allospecific vocal learning by grey parrots (Psittacus erithacus).

[41] Tomas Persson and others. 2018. Spontaneous cross-species imitation in interactions between chimpanzees and zoo visitors.

[42] María Pifarré and others. 2012. The effect of zoo visitors on the behaviour and faecal cortisol of the Mexican wolf. 
[43] Patricia Pons and others. 2016. Sound to your objects: a novel design approach to evaluate orangutans' interest in sound-based stimuli. In ACI'16. ACM.

[44] Sandra Quadros and others. 2014. Zoo visitor effect on mammal behaviour: Does noise matter?

[45] Nikki S Rickard and others. 2005. The effect of music on cognitive performance: Insight from neurobiological and animal studies.

[46] Sarah Elizabeth Ritvo. 2013. Music preference and discrimination in three Sumatran orangutans.

[47] Becky Scheel. 2018. Designing digital enrichment for orangutans. In ACI'18. ACM.

[48] David Sibley. 2011. The Proper Use of Playback in Birding. https://www. sibleyguides.com/2011/04/ the-proper-use-of-playback-in-birding/

[49] Marek Špinka, Françoise Wemelsfelder, and others. 2011. Environmental challenge and animal agency, 27-43.

[50] Stephanie Spinner. 2012. Alex the parrot: no ordinary bird. Knopf Books for Young Readers.

[51] Austin Stewart. 2015. Second Livestock. http://www . theaustinstewart. com/secondlivestock.html

[52] Thomas Suddendorf and Andrew Whiten. 2001. Mental evolution and development: Evidence for secondary representation in children, great apes, and other animals.

[53] Dietmar Todt. 1975. Social learning of vocal patterns and modes of their application in grey parrots.

[54] K Uetake and others. 1997. Effect of music on voluntary approach of dairy cows to an automatic milking system.
[55] Elaine N Videan and others. 2007. Effects of two types and two genre of music on social behavior in captive chimpanzees (Pan troglodytes).

[56] Lisa J Wallis and others. 2017. Utilising dog-computer interactions to provide mental stimulation in dogs especially during ageing. In $A C I ' 17$. ACM.

[57] Samantha J Ward and Vicky Melfi. 2015. Keeper-animal interactions: Differences between the behaviour of zoo animals affect stockmanship.

[58] Sarah Webber and others. 2016. HCI Goes to the Zoo:. In CHI'16.

[59] Sarah Webber and others. 2017. Kinecting with orangutans: Zoo visitors' empathetic responses to animals? use of interactive technology. In CHI'17.

[60] Deborah L Wells. 2009. The effects of animals on human health and well-being, 523-543.

[61] Deborah L Wells and Rosie M Irwin. 2008. Auditory stimulation as enrichment for zoo-housed Asian elephants (Elephas maximus).

[62] Michelle Westerlaken and Stefano Gualeni. 2014. Felino: The philosophical practice of making an interspecies videogame. In The Philosophy of Computer Games.

[63] Isabelle Williams and others. 2017. The effect of auditory enrichment, rearing method and social environment on the behavior of zoo-housed psittacines (Aves: Psittaciformes); implications for welfare.

[64] Robert Yanofsky and others. 1978. Changes in general behavior of two mandrills (Papio sphinx) concomitant with behavioral testing in the zoo. 